\title{
The Prognostic Value of a Validated and Automated Intravascular Ultrasound-Derived Calcium Score
}

\author{
Tara Neleman $^{1} \cdot$ Shengnan Liu $^{1}$ - Maria N. Tovar Forero ${ }^{1}$ - Eline M. J. Hartman ${ }^{1}$ - Jurgen M. R. Ligthart ${ }^{1}$. \\ Karen T. Witberg ${ }^{1} \cdot$ Paul Cummins $^{1} \cdot$ Felix Zijlstra ${ }^{1} \cdot$ Nicolas M. Van Mieghem $^{1} \cdot$ Eric Boersma $^{1} \cdot$ Gijs van Soest $^{1}$. \\ Joost Daemen $^{1}$ (D)
}

Received: 28 September 2020 / Accepted: 11 January 2021 / Published online: 23 February 2021

(C) The Author(s) 2021

\section{Abstract}

Background Coronary calcification has been linked to cardiovascular events. We developed and validated an algorithm to automatically quantify coronary calcifications on intravascular ultrasound (IVUS). We aimed to assess the prognostic value of an IVUS-calcium score (ICS) on patient-oriented composite endpoint (POCE).

Methods We included patients that underwent coronary angiography plus pre-procedural IVUS imaging. The ICS was calculated per patient. The primary endpoint was a composite of all-cause mortality, stroke, myocardial infarction, and revascularization (POCE).

Results In a cohort of 408 patients, median ICS was 85 . Both an ICS $\geq 85$ and a 100 unit increase in ICS increased the risk of POCE at 6-year follow-up (adjusted hazard ratio (aHR) 1.51, 95\%CI 1.05-2.17, $p$ value $=0.026$, and aHR 1.21, 95\%CI 1.04 $1.41, p$ value $=0.014$, respectively).

Conclusions The ICS, calculated by a validated automated algorithm derived from routine IVUS pullbacks, was strongly associated with the long-term risk of POCE.

Keywords Coronary artery disease $\cdot$ Coronary calcification $\cdot$ Intravascular ultrasound $\cdot$ Automated quantification $\cdot$ Prognosis

$\begin{array}{ll}\text { Abbreviations } \\ \text { aHR } & \text { Adjusted hazard ratio } \\ \text { BMS } & \text { Bare metal stent } \\ \text { BRS } & \text { Bioresorbable scaffold } \\ \text { CABG } & \text { Coronary artery bypass grafting } \\ \text { CI } & \text { Confidence interval } \\ \text { ICS } & \text { IVUS-calcium score } \\ \text { IQR } & \text { Interquartile range } \\ \text { IVUS } & \text { Intravascular ultrasound } \\ \text { OCT } & \text { Optical coherence tomography } \\ \text { PCI } & \text { Percutaneous coronary intervention } \\ \text { POCE } & \text { Patient-oriented composite endpoint } \\ \text { SD } & \text { Standard deviation }\end{array}$

Editor-in-Chief Enrique Lara-Pezzi oversaw the review of this article

Joost Daemen

j.daemen@erasmusmc.nl

1 Department of Cardiology, Thoraxcenter, Erasmus University Medical Center, Rotterdam, The Netherlands

\section{Introduction}

Calcification is the end result of apoptosis of smooth muscle cells and macrophages and proved to be associated with advanced stages of atherosclerosis [1]. Vascular calcification is an established independent predictor of all-cause mortality and cardiovascular events in a general population [2].

The coronary artery calcium score as detected by computed tomography emerged as the strongest predictor of incident coronary artery disease in an asymptomatic population [3-7]. Besides its predictive value, the presence and extent of coronary calcium are also of importance in light of percutaneous coronary intervention (PCI). Presence of coronary calcifications is considered to be a major predictor of suboptimal stent expansion, which in turn increases the risk for target vessel failure [8-10].

Coronary angiography lacks sensitivity and precludes quantification of the extent of coronary calcium. Intravascular imaging techniques like intravascular ultrasound (IVUS) and optical coherence tomography (OCT) have a higher sensitivity to detect coronary calcification but lack the ability to 
automatically quantify calcium burden $[11,12]$. While several algorithms have been assessed to automatically detect and quantify the extent of coronary calcification, there is a lack of data linking validated IVUS-derived calcium scores to clinical outcome [13-15].

We recently developed, trained, and validated a novel algorithm to automatically detect and quantify coronary artery calcium on IVUS using machine learning techniques [16]. The objective of the present study is to assess the clinical applicability of this algorithm to determine patient outcomes in a large local PCI and IVUS registry.

\section{Methods}

\section{Inclusion and Exclusion}

In this retrospective single-center cohort study, we enrolled patients who underwent coronary angiography with preprocedural IVUS imaging. We screened patients for eligibility between January 2008 and January 2018. Patients were included if motorized IVUS pullbacks over a length $\geq 40 \mathrm{~mm}$ in a native coronary artery were available. IVUS-related exclusion criteria were (1) the presence of stent struts, (2) poor imaging quality, and (3) catheter in false lumen. Only one pullback per patient was included; in case of availability of pullbacks from different coronary arteries, analyses were restricted to the longest analyzable pullback. Also, in case of multiple pullbacks from the same artery, analyses were restricted to the longest pullback.

\section{Index Procedure}

Invasive studies evolved per standard practice and IVUS imaging was used according to operator's preference. IVUS imaging was performed with a $40 \mathrm{MHz}$ IVUS catheter (Atlantis SR Pro2 or OptiCross, Boston Scientific Corporation, Natick, Mass) at a pullback speed of $0.5 \mathrm{~mm} / \mathrm{s}$. IVUS pullbacks were stored as dicoms in a dedicated local database and were analyzed offline.

\section{IVUS-Calcium Score}

We recently developed and validated an automatic calcium detection algorithm to quantify coronary calcium on IVUS pullbacks [16]. Coronary calcium as visualized by IVUS was defined as a sharp white border accompanied by a dark acoustic shadowing [17]. A support vector machine was trained and tested on 35 pullbacks with manually annotated calcium arcs to detect this feature of calcium automatically per A-line in each frame. Reported accuracy, precision, and recall were $0.89,0.92$, and 0.85 , respectively [16]. The IVUScalcium score (ICS) was computed per pullback and defined as the number of calcium-positive A-lines divided by the total numbers of A-lines times 1000.

\section{Data Collection Procedure}

The primary endpoint was Academic Research Consortium-2 defined Patient-Oriented Composite Endpoint (POCE): a composite endpoint consisting of all-cause mortality, any stroke, any myocardial infarction, and any revascularization [18]. Secondary endpoints included target vessel revascularization, target vessel myocardial infarction, and individual components of POCE. Target vessel was defined as the vessel that was imaged with IVUS during the index procedure and that was used for computation of the ICS: the study vessel. Survival data were obtained through municipal civil registry checks. Data on outcome measures and baseline characteristics were obtained from local electronic patient records whenever available. A health questionnaire was sent to all living patients evaluating re-admission and cardiovascular and cerebrovascular events. Patient-reported outcomes were verified with source documentation from referring hospitals. General practitioners, referring cardiologists, and patients were contacted as necessary for additional information. Ethical approval for this study was waived by the Institutional Review Board of the Erasmus Medical Center because of the retrospective nature of the clinically collected data.

\section{Data Analysis}

Normality of continuous variables was tested using the ShapiroWilk test. Normally distributed variables are displayed as mean \pm standard deviation (SD), whereas non-normally distributed variables are displayed as median and interquartile range (IQR; 25th-75th percentile). Categorical variables are displayed as counts and percentages. In this first clinical validation study, we decided to evaluate the ICS both as a categorical and a continuous variable. The ICS was dichotomized according to the median of 85. Differences in baseline continuous variables between patients with an ICS $<85$ and an ICS $\geq 85$ were compared with the independent Student's $t$ test or the MannWhitney $U$ test, and differences in categorical covariates between patients with an ICS $<85$ and an ICS $\geq 85$ were evaluated with the Pearson's chi-square test or Fischer's exact test, as appropriate. We performed uni- and multivariate linear regression to identify factors associated with ICS. Because residuals of regression analyses using the ICS directly were non-normally distributed, we applied a square root transformation to the ICS ( $\sqrt{ } \mathrm{ICS})$ for this analysis (standard log transformation also did not meet linear regression assumptions). Baseline patient characteristics with $p$ value $<0.1$ in univariate linear regression for $\sqrt{\mathrm{ICS}}$ were included in the full multivariate model.Patients lost to follow-up were considered being at risk for the event until 6 years (2191 days) after the index procedure or until the day of 
the last contact, at which point they were censored. In case of multiple events, the first event was taken into account. The Kaplan-Meier method was used to estimate survival functions for patients with ICS $<85$ and ICS $\geq 85$. Differences in survival time distributions between these groups of patients were assessed using the log-rank test. Uni- and multivariate Cox regression models were built to study the relationship between ICS and the study endpoints. Multivariate adjustment was carried out to remove potential bias introduced by confounding variables and other influential factors. Selection of variables for multivariate adjustment in each model was performed according to clinical relevance in literature and presence of a univariate association in our own results. All covariates were checked for satisfying the proportional hazard assumption by Schoenfeld residual tests. Competing risks were taken into account by using the cumulative incidence function to calculate cumulative incidences and cause-specific hazard regression to obtain hazard ratios. A two-sided $p$ value of $<0.05$ was considered as statistically significant. Statistical analyses were performed using IBM SPSS Statistics for Windows, version 25.0 (IBM Corp., Armonk, NY, USA) and R (R Core Team 2019; version 3.5.2, packages: ggplot2, survival, splines, cmprsk).

\section{Results}

\section{Screening and Inclusion}

Between January 2008 and January 2018, 1265 IVUS pullbacks were identified from 892 patients undergoing coronary angiography plus pre-procedural IVUS imaging. In 347 pullbacks, stent struts from previously implanted stents were present, and 355 pullbacks were too short $(<40 \mathrm{~mm})$. In total, 408 patients were included in the analysis. Detailed information on the number of exclusions can be found in the Electronic Supplementary Material (Supplementary Table 1).

\section{Baseline Characteristics}

The median age of the cohort was 65.7 years (IQR 57.2-72.2) and $72.5 \%$ were male. Stable angina was the presenting symptom in $56.4 \%$ and $86.5 \%$ of patients underwent subsequent revascularization (PCI $(n=334 ; 81.9 \%)$ or coronary artery bypass grafting (CABG) $(n=19 ; 4.7 \%))$ (Fig. 1). Renal impairment (eGFR $<60 \mathrm{ml} / \mathrm{min}$ ) was present in $18.9 \%$ of patients.

The median ICS was 85 (IQR 25-169) and ranged from 0 to 503 (Fig. 2). Patients with an ICS $\geq 85$ were older, more frequently suffered from hypertension and hypercholesterolemia, more often had a previous stroke, peripheral artery disease, and renal impairment, and were more often referred for CABG. Patients with an ICS $<85$ more often presented with acute coronary syndromes (Table 1).
A total of $308(75.5 \%)$ vessels were subsequently treated with either PCI $(n=289 ; 70.1 \%)$ or CABG $(n=19 ; 4.7 \%)$ (Fig. $1)$. The remaining 100 vessels $(24.5 \%)$ were a mix of nonculprit coronary arteries $(n=45)$ and vessels from patients that were finally treated conservatively $(n=55)$. Study vessels with an ICS $\geq 85$ were more often revascularized compared with vessels with an ICS $<85$ (Table 1). The distribution of study vessels was similar between the groups.

A total of $57.1 \%$ (233/408) patients were on statin therapy at the time of the index procedure. Patients on statin treatment had significantly higher ICS as compared with statin naive patients $(121 \pm 101.4$ vs $91 \pm 103.2, p=0.006)$ and patients with ICS $\geq 85$ appeared to be more often on statin therapy as compared with patients with ICS $<85(66.1 \%$ vs $54.4 \%, p=$ $0.018)$.

\section{Multivariate Linear Regression}

Median $\sqrt{\text { ICS was }} 9.2$ (IQR 5.1-15.7) and ranged from 0 to 22.4. Increasing age and previous stroke remained as significant explanatory variables for $\sqrt{ }$ ICS in the multivariate model. Previous stroke was associated with a mean difference of 2.46 units of $\operatorname{ICS}(p=0.029)$, whereas each year of increase in age was associated with a mean increase of 0.07 units of $\sqrt{ } \operatorname{ICS}(p=$ 0.002) (Table 2). See Supplementary Figure 1 (Electronic Supplementary Material) for a visual interpretation of the significant covariates when back-transformed to the linear scale.

\section{Survival Analysis}

The median follow-up was 2166 (987-3299) days. During a follow-up of 6 years (2191 days), POCE occurred in 152 patients (cumulative incidence $44.4 \%$ ). The cumulative incidence of POCE for patients with ICS $\geq 85$ was $51.8 \%$ versus $36.4 \%$ for patients with ICS $<85$ ( $p=0.008$ ) (Fig. 3).

Both an ICS $\geq 85$ and each 100 units increase in the ICS were associated with a significant increase in the risk of POCE: the adjusted hazard ratio (aHR) for ICS $\geq 85$ was $1.51(95 \%$ CI $1.05-2.17, p$ value $=0.026)$ and for each 100 units increase in ICS 1.21 (95\% CI 1.04-1.41, $p$ value $=$ 0.014) (Table 3). A 100 unit increase in the ICS appeared to be associated with a significantly increased rate of target vessel revascularization: the aHR for each 100 units increase in ICS was 1.37 (95\% CI 1.06-1.77, $p$ value $=0.017)$. Detailed multivariate models for the prognostic value of the ICS on the individual components of POCE can be found in Supplementary Table 2 (Electronic Supplementary Material).

In the study vessel revascularized at index cohort $(n=308)$ and study vessel not revascularized at index cohort $(n=100)$, the cumulative incidences of POCE for patients with ICS $\geq 85$ versus patients with ICS $<85$ were $50.8 \%$ versus $38.0 \%$ ( $p=$ $0.064)$ and $56.0 \%$ versus $32.3 \%(p=0.028)$ (Fig. 3). 


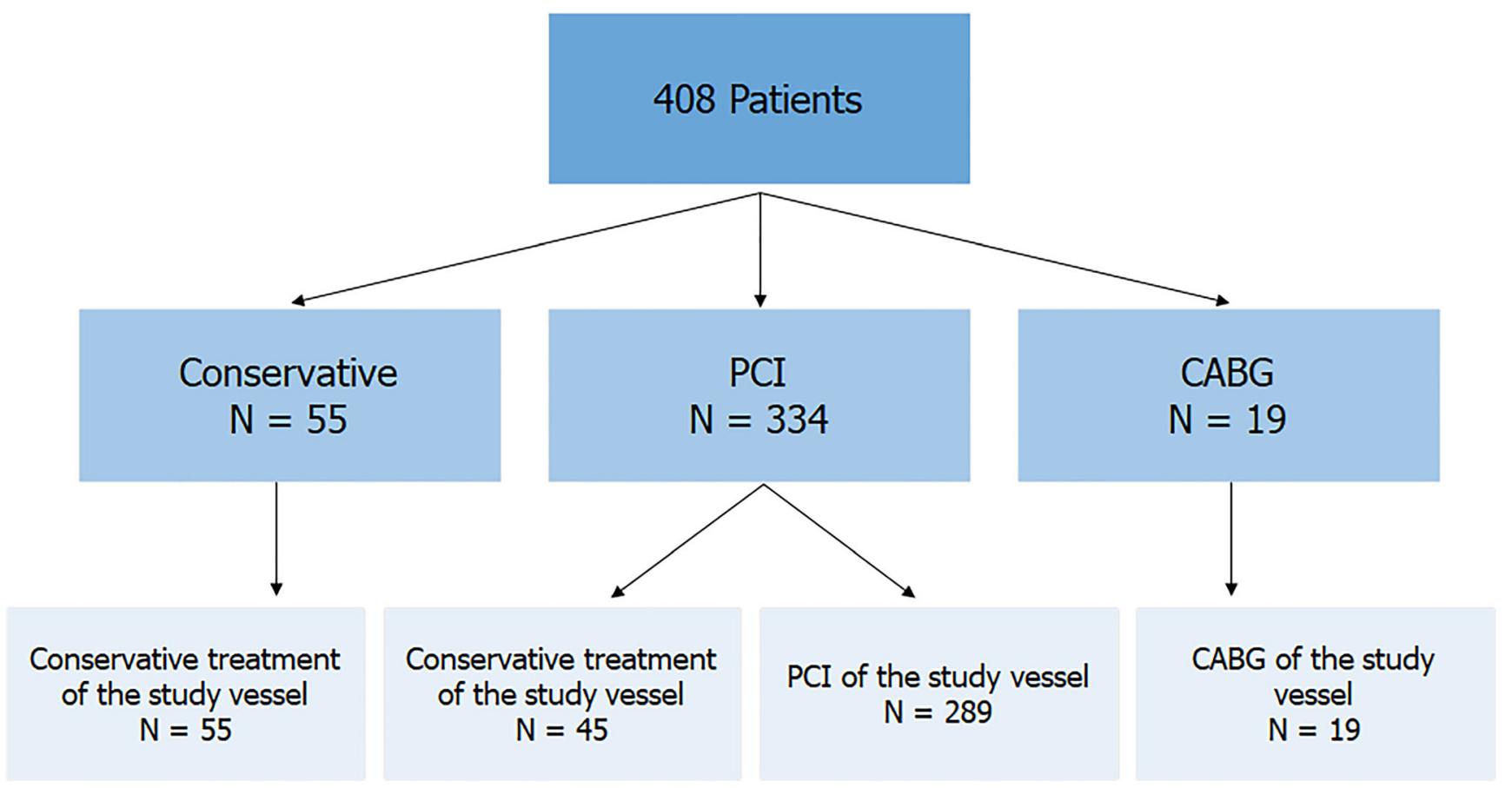

Fig. 1 Flowchart indicating type of treatment following enrolment. Abbreviations: CABG = coronary artery bypass graft, IVUS = intravascular ultrasound, $\mathrm{PCI}=$ percutaneous coronary intervention

\section{Discussion}

In the present study, we assessed the prognostic value of a validated and automated IVUS-derived calcium score, the ICS, on POCE at 6 years. We demonstrated that (1) patients with an ICS $\geq 85$ had an overall higher cardiovascular risk profile and (2) the ICS both as a dichotomized (ICS $\geq 85$ ) and as a continuous score significantly predicts POCE.

Apoptosis of smooth muscle cells and macrophages are believed to induce the formation of microcalcifications ( 0.5 $15 \mu \mathrm{m})$ in the intimal layer of the coronary artery $[19,20]$. Fusion of these microcalcifications and further progression of calcification over time results in calcified plaques formed by calcified sheets or plates that can be identified with computed tomography and during invasive coronary angiography. Mildly calcified plaques are common and moderate to severe calcifications can be found in up to $18 \%$ of patients with an inherent risk for increased event rates [21].

Our findings support the evidence that patients with a greater extent of coronary calcification have a higher cardiovascular risk profile [6,21-24]. We found that patients with an ICS $\geq 85$ were more likely to be older, have hypercholesterolemia, hypertension, renal impairment, a previous stroke, previous peripheral arterial disease, present with stable angina (as compared with
Fig. 2 The frequency distribution of ICS. Median ICS was 85 (IQR 25-169) and ranged from 0 to 503. Abbreviations: ICS $=$ IVUScalcium score, $\mathrm{IQR}=$ interquartile range, IVUS = intravascular ultrasound

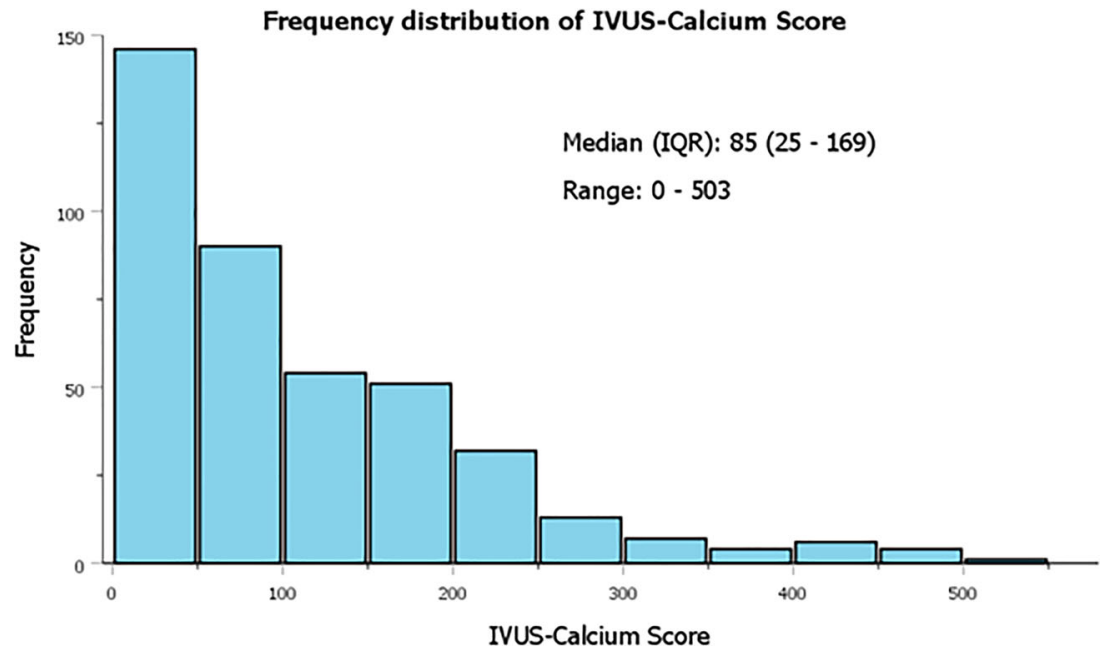


Table 1 Baseline characteristics of total cohort, patients with an ICS $<85$ and ICS $\geq 85$. Values are displayed as median (IQR) or $n(\%)$

\begin{tabular}{|c|c|c|c|c|}
\hline & $\begin{array}{l}\text { All patients } \\
N=408\end{array}$ & $\begin{array}{l}\text { ICS }<85 \\
N=204\end{array}$ & $\begin{array}{l}\text { ICS } \geq 85 \\
N=204\end{array}$ & $P$ value \\
\hline \multicolumn{5}{|l|}{ Patient level } \\
\hline Age in years & $65.7(57.2-72.2)$ & $62.7(53.9-70.2)$ & $68.0(61.1-74.2)$ & $<0.001$ \\
\hline Male gender & $296(72.5 \%)$ & $147(72.1 \%)$ & $149(73.0 \%)$ & 0.82 \\
\hline Hypertension & $260(63.7 \%)$ & $115(56.4 \%)$ & $145(71.1 \%)$ & 0.002 \\
\hline Hypercholesterolemia & $226(55.4 \%)$ & $96(47.1 \%)$ & $130(63.7 \%)$ & 0.001 \\
\hline Statin treatment & $233(57.1 \%)$ & $106(54.4 \%)$ & $127(66.1 \%)$ & 0.018 \\
\hline Diabetes mellitus & $84(20.6 \%)$ & $34(16.7 \%)$ & $50(24.5 \%)$ & 0.050 \\
\hline Positive familial history & $152(37.3 \%)$ & $80(39.2 \%)$ & $72(35.3 \%)$ & 0.41 \\
\hline Smoking & $165(40.4 \%)$ & $86(42.2 \%)$ & $79(38.2 \%)$ & 0.48 \\
\hline Previous MI & $104(25.5 \%)$ & $52(25.5 \%)$ & $52(25.5 \%)$ & 1 \\
\hline Previous PCI & $106(26.1 \%)$ & $53(26.0 \%)$ & $53(26.0 \%)$ & 1 \\
\hline Previous CABG & $16(3.9 \%)$ & $7(3.4 \%)$ & $9(4.4 \%)$ & 0.61 \\
\hline Previous stroke & $22(5.4 \%)$ & $4(2.0 \%)$ & $18(8.8 \%)$ & 0.003 \\
\hline Previous PAD & $42(10.3 \%)$ & $13(6.4 \%)$ & $29(14.2 \%)$ & 0.006 \\
\hline $\begin{array}{l}\text { Renal impairment } \\
\quad(\text { eGFR }<60 \mathrm{ml} / \mathrm{min})\end{array}$ & $77(18.9 \%)$ & $26(13.7 \%)$ & $51(25.8 \%)$ & 0.003 \\
\hline eGFR (ml/min) & $79(63-90)$ & $83(68-93)$ & $74(58-88)$ & $<0.001$ \\
\hline Presentation with ACS & $178(43.6 \%)$ & $103(50.5 \%)$ & $75(36.8 \%)$ & 0.005 \\
\hline Index treatment & & & & 0.005 \\
\hline PCI performed at baseline & $334(81.9 \%)$ & $169(82.8 \%)$ & $165(80.9 \%)$ & \\
\hline CABG performed at baseline & $19(4.7 \%)$ & $3(1.5 \%)$ & $16(7.8 \%)$ & \\
\hline Conservative treatment at baseline & $55(13.5 \%)$ & $32(15.7 \%)$ & $23(11.3 \%)$ & \\
\hline \multicolumn{5}{|l|}{ Vessel level } \\
\hline Study vessel & & & & 0.13 \\
\hline Left anterior descending & $262(64.2 \%)$ & $123(60.3 \%)$ & $139(68.1 \%)$ & \\
\hline Left circumflex & $59(14.5 \%)$ & $33(16.2 \%)$ & $26(12.7 \%)$ & \\
\hline Right coronary artery & $77(18.9 \%)$ & $45(22.1 \%)$ & $32(15.7 \%)$ & \\
\hline Left main stem & $10(2.5 \%)$ & $3(1.5 \%)$ & $7(3.4 \%)$ & \\
\hline $\begin{array}{l}\text { Study vessel revascularized at index } \\
\text { procedure (either PCI or CABG) }\end{array}$ & $308(75.5 \%)$ & $144(71.1 \%)$ & $164(80.9 \%)$ & 0.021 \\
\hline
\end{tabular}

$A C S$ acute coronary syndrome, $B M S$ bare metal stent, $B R S$ bioresorbable scaffold, $C A B G$ coronary artery bypass graft, $D E S$ drug-eluting stent, $e G F R$ estimated glomerular filtration rate, ICS IVUS-calcium score, IQR interquartile range, IVUS intravascular ultrasound, $M I$ myocardial infarction, $P A D$ peripheral artery disease, $P C I$ percutaneous coronary intervention acute coronary syndrome), and undergo subsequent coronary revascularization. After multivariate adjustment, age and previous cerebrovascular accident remained independent predictors for ICS, which is presumably due to our relatively small sample size. Several studies have demonstrated that the coronary artery calcium score is a strong predictor for cardiovascular events in asymptomatic individuals $[2-4,6,25,26]$. To the best of our knowledge, the ICS is the first validated score for coronary artery calcification linked to cardiovascular events since the introduction of the computed tomography-derived coronary artery calcium score 30 years ago [5]. In fact, we found a $51 \%$ increased risk of POCE in patients with an ICS above the median of 85. The fact that we have found that a calcium score based on one coronary artery alone is associated with impaired patient outcome reflects the systematic nature of atherosclerotic cardiovascular disease. Caution is warranted when comparing the computer tomography-derived coronary artery calcium score and the ICS. The non-invasively obtained coronary artery calcium score has its particular value in decision-making in cardiovascular risk assessment, in particular in the asymptomatic population, while the ICS has been derived from invasive intracoronary evaluation in patients with suspected coronary artery disease [27].

An apparent heterogeneity was found in the predictive value of an ICS (cut-off 85) for POCE in vessels that were target of revascularization versus non-culprit vessels (Fig. 3). The latter could be explained by a higher ICS in vessels that were target to revascularization as compared with non-culprit vessels (median ICS 91 versus median ICS 64, respectively). Moreover, higher ICS (each 100 units increase) proved to be an independent predictor of target vessel revascularization. Nevertheless, these 
Table 2 Uni- and multivariate linear regression models for $\sqrt{ } \mathrm{ICS}$

\begin{tabular}{|c|c|c|c|c|c|c|}
\hline \multirow[t]{2}{*}{ Linear regression $\sqrt{\mathrm{ICS}}$} & \multicolumn{3}{|c|}{ Univariate } & \multicolumn{3}{|c|}{ Multivariate } \\
\hline & $\beta$ & $95 \% \mathrm{CI} \beta$ & $P$ value & $\beta$ & $95 \%$ CI $\beta$ & $P$ value \\
\hline Gender & 0.22 & $-0.90-1.34$ & 0.70 & & & \\
\hline Age, in years & 0.11 & $0.07-0.15$ & $<0.001$ & 0.07 & $0.03-0.12$ & 0.002 \\
\hline Hypertension & 1.65 & $0.63-2.68$ & 0.002 & 0.89 & $-0.18-1.96$ & 0.10 \\
\hline Hypercholesterolemia & 1.53 & $0.54-2.53$ & 0.002 & 0.92 & $-0.11-1.94$ & 0.079 \\
\hline Diabetes mellitus & 1.52 & $0.30-2.75$ & 0.015 & 1.02 & $-0.19-2.22$ & 0.097 \\
\hline Positive familial history & -0.62 & $-1.65-0.41$ & 0.24 & & & \\
\hline Smoking & -0.05 & $-1.07-0.97$ & 0.92 & & & \\
\hline Previous MI & 0.04 & $-1.10-1.19$ & 0.94 & & & \\
\hline Previous PCI & 0.23 & $-0.91-1.36$ & 0.70 & & & \\
\hline Previous CABG & 1.15 & $-1.42-3.72$ & 0.38 & & & \\
\hline Previous stroke & 3.87 & $1.69-6.05$ & 0.001 & 2.46 & $0.25-4.68$ & 0.029 \\
\hline Previous PAD & 2.37 & $0.74-4.00$ & 0.004 & 1.10 & $-0.54-2.74$ & 0.19 \\
\hline eGFR & -0.04 & -0.06 to -0.02 & 0.001 & -0.02 & $-0.04-0.01$ & 0.25 \\
\hline Presentation with ACS & -1.35 & -2.35 to -0.35 & 0.008 & -0.87 & $-1.88-0.14$ & 0.091 \\
\hline Study vessel LAD & 0.43 & $-0.61-1.47$ & 0.42 & & & \\
\hline
\end{tabular}

$A C S$ acute coronary syndrome, $C A B G$ coronary artery bypass graft, $C I$ confidence interval, $e G F R$ estimated glomerular filtration rate, ICS IVUS-calcium score, $L A D$ left anterior descending, $M I$ myocardial infarction, $P A D$ peripheral artery disease, $P C I$ percutaneous coronary intervention findings must be considered as explorative and hypothesisgenerating as our sample size was too small to (adequately) control for confounding variables in these analyses.

Intravascular imaging using either IVUS or OCT has proven to be superior to coronary angiography and multi-slice computed tomography for the detection of coronary calcification [11, $12,28]$. Non-automated assessment of coronary calcium for both clinical and research purposes is time-consuming. Recently, several algorithms have been proposed to automatically detect plaque features in intravascular imaging techniques. The concept of virtual histology (VH)-IVUS was first introduced in 2002 and demonstrated to be able to detect plaque characteristics in non-culprit coronary arteries that predict major adverse cardiovascular events [29-31]. At present, the use of VH-IVUS has practically been abandoned due to repetitive questions on the validity of the algorithm and lack of ability of VH-IVUS to alter patient management.Previous automated classification tools for detection of calcium in $20 \mathrm{MHz}$ IVUS pullbacks and OCT have been proposed as well, but have mainly focused on tissue type segmentation in cross-sectional images $[13,14,32]$. This study is the first to derive a clinically relevant score and to validate this score against clinical outcome.

On a patient level, the automatic detection of coronary calcification might improve our ability to classify the risk of an individual patient. Next to presentation with ACS, a history of CABG, and eGFR, ICS proved to be among the strongest predictors of POCE at 6 years. On a lesion level, the ICS might play an important role in future algorithms for lesion preparation in an era where the treatment armamentarium for calcified coronary artery disease is rapidly expanding. The direct relation between lesion-specific ICS and stent expansion might shed further light on this issue and is therefore subject of further study. Another potential application of the ICS includes serial plaque imaging assessing the effect of pharmacological interventions on calcified plaque volume.

At present, the use of the ICS is restricted to patients referred for coronary angiography in which IVUS catheters from vendors in which the algorithm has been validated are used. Moreover, the use of the ICS is restricted to native coronary artery disease and vessels that have not been subject to prior stenting. Dedicated future studies towards the feasibility of implementation of our algorithm in commercially available IVUS systems are needed which limits current clinical use. Nevertheless, as mentioned above, several clinical scenarios could be envisaged in which the ICS could be of use in both clinical practice as well as research settings.

\section{Limitations}

Strengths of our study include the access to a large local IVUS and PCI database and a validated algorithm built to detect specific A-line features and automatically generate a calcium score. Given the lack of a need for manual contour detection, the algorithm provides no data on atheroma volume precluding any statements on the prognostic utility of ICS over plaque burden. Moreover, given the inability of IVUS to assess calcium thickness, the ICS is not based on total calcium volume in the coronary artery, but on calcium arc and length only.

Secondly, strict inclusion criteria and retrospective screening led to a limited sample size, which potentially impacted 
Fig. 3 Cumulative incidence curves for POCE for the total cohort (a), the subgroup of patients in whom the study vessel was revascularized (b), and the subgroup of patients in whom the study vessel was not revascularized $(\mathbf{c})$. The red solid lines represent the cumulative incidence curves for patients with an ICS $\geq 85$ and the dashed blue lines represent the cumulative incidence curves for patients with an ICS $<85$. The cumulative incidence curves of patients with an ICS $\geq 85$ versus ICS $<85$ were compared using the log-rank test. Abbreviations: ICS $=$ IVUScalcium score, $\mathrm{POCE}=$ patientoriented composite endpoint a

Total cohort $(n=408)$

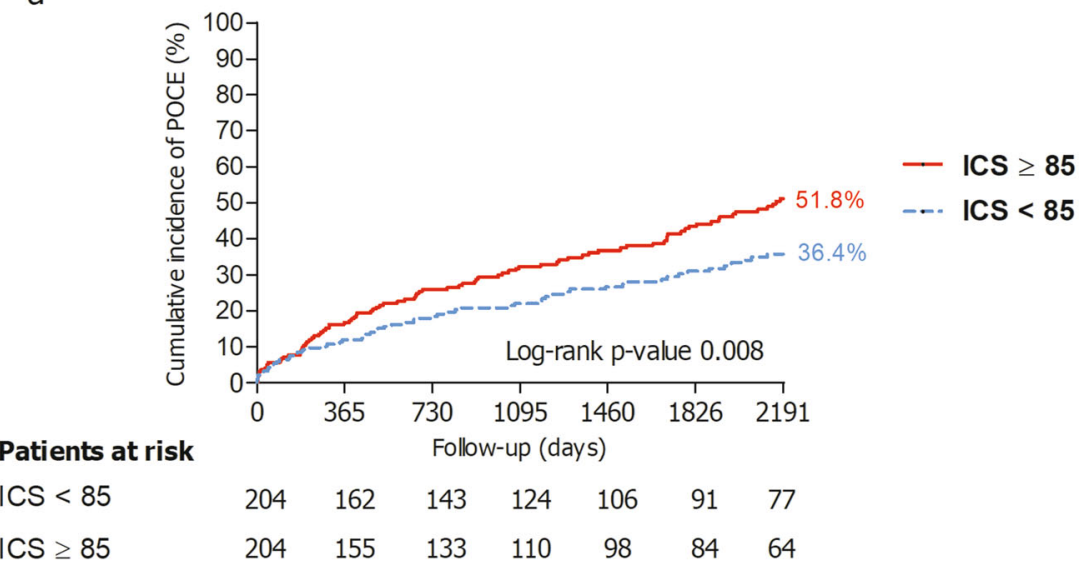

C

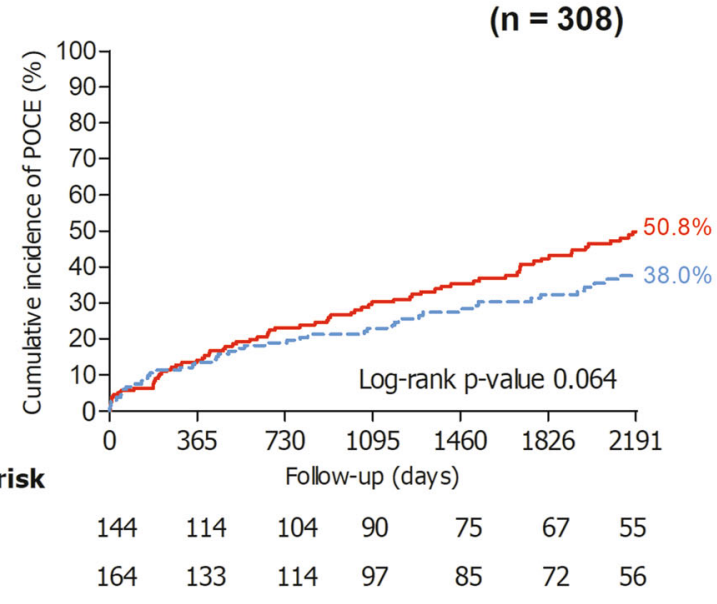

ICS in vessels not revascularized at index $(n=100)$

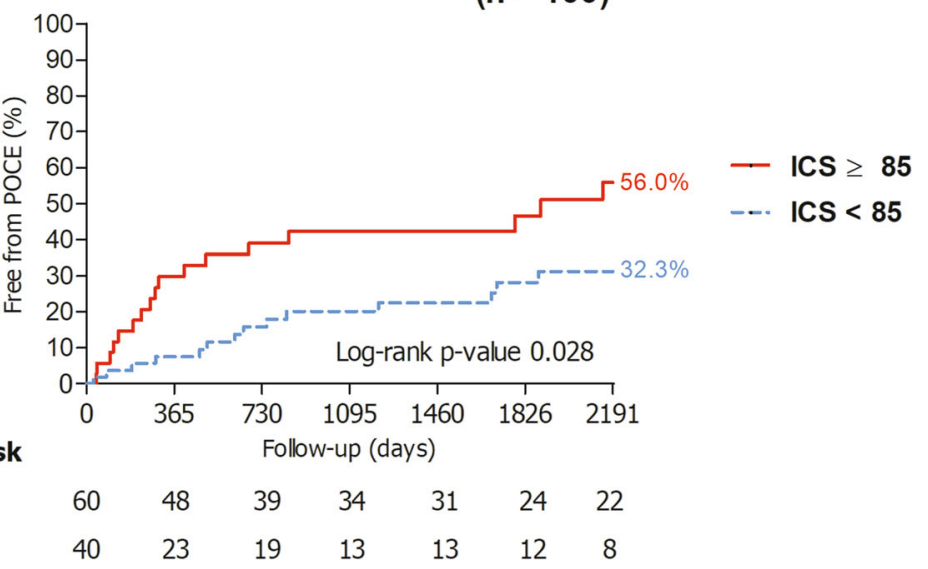

the scope of the present analysis. Thirdly, we decided to dichotomize the ICS according to its median value in this first clinical validation study, similar to previous published work on the lipid core burden index [33]. This does not imply that an ICS $\geq 85$ is the best cut-off value. Finally, while ICS was significantly higher in patients taking statins, we could not ascertain a definite causal relation and exclude the fact that the relation found was a reflection of the higher overall risk 
Table 3 Event rates and multivariate Cox regression models for the composite endpoint POCE and the individual endpoints of POCE for both ICS $\geq 85$ and ICS as a continuous covariate ( ICS $_{100}$ units). Values are $n$ (cumulative incidence) unless specified otherwise. The multivariate model for POCE was stratified on diabetes due to non-proportional hazards. Detailed information and tables of full models can be found in Supplementary Table 2 (Electronic Supplementary Material)

\begin{tabular}{|c|c|c|c|c|c|c|c|}
\hline \multirow[b]{2}{*}{ Event of interest } & \multicolumn{3}{|c|}{ Number of patients with event at 6-year follow-up } & \multicolumn{2}{|l|}{$\mathrm{ICS} \geq 85$} & \multicolumn{2}{|l|}{$\mathrm{ICS}_{100 \text { units }}$} \\
\hline & Total $(n=408)$ & ICS < $85(n=204)$ & $\mathrm{ICS} \geq 85(n=204)$ & $\mathrm{HR}(95 \% \mathrm{CI})$ & $p$ value & HR $(95 \%$ CI) & $p$ value \\
\hline POCE & $152(44.4 \%)$ & $61(36.4 \%)$ & $91(51.8 \%)$ & $1.51(1.05-2.17)$ & 0.026 & $1.21(1.04-1.41)$ & 0.014 \\
\hline Any revascularization & $80(23.2 \%)$ & $37(22.1 \%)$ & $43(24.2 \%)$ & $1.31(0.82-2.10)$ & 0.26 & $1.23(1.00-1.51)$ & 0.055 \\
\hline PCI & $71(20.8 \%)$ & $34(20.4 \%)$ & $37(21.1 \%)$ & $1.25(0.77-2.05)$ & 0.37 & $1.20(0.96-1.49)$ & 0.11 \\
\hline CABG & $13(3.6 \%)$ & $5(2.9 \%)$ & $8(4.3 \%)$ & $1.61^{\mathrm{a}}(0.53-4.91)$ & 0.41 & $1.30^{\mathrm{a}}(0.82-2.06)$ & 0.27 \\
\hline Target vessel revascularization & $42(12.0 \%)$ & $18(10.4 \%)$ & $24(13.4 \%)$ & $1.68(0.88-3.19)$ & 0.11 & $1.37(1.06-1.77)$ & 0.017 \\
\hline Any myocardial infarction & $31(9.3 \%)$ & $12(7.3 \%)$ & $19(11.0 \%)$ & $1.44(0.69-3.02)$ & 0.33 & $1.28(0.95-1.73)$ & 0.10 \\
\hline Target vessel myocardial infarction & $20(5.7 \%)$ & $8(4.5 \%)$ & $12(6.7 \%)$ & $1.47(0.60-3.60)$ & 0.40 & $1.29(0.90-1.85)$ & 0.17 \\
\hline Any stroke & $14(4.1 \%)$ & $5(2.9 \%)$ & $9(5.3 \%)$ & $1.80^{\mathrm{a}}(0.60-5.38)$ & 0.29 & $1.37^{\mathrm{a}}(0.89-2.10)$ & 0.15 \\
\hline All-cause mortality & $68(18.9 \%)$ & $21(12.0 \%)$ & $47(25.6 \%)$ & $1.55(0.91-2.64)$ & 0.10 & $1.16(0.94-1.43)$ & 0.17 \\
\hline
\end{tabular}

$C A B G$ coronary artery bypass graft, $C I$ confidence interval, $H R$ hazard ratio, ICS IVUS-calcium score, $P C I$ percutaneous coronary intervention, $P O C E$ patient-oriented composite endpoint

${ }^{\text {a }}$ Due to a limited number of events, only univariate cox regression could be provided

profile in patients with a higher ICS. Yet, despite these limitations, we could demonstrate that the ICS significantly increases the risk of cardio-cerebrovascular events.

\section{Conclusion}

The ICS is a novel scoring tool that automatically quantifies the extent of coronary calcification on IVUS and is significantly related to impaired cardiovascular and cerebrovascular outcomes in an all-comer population of patients undergoing coronary angiography and IVUS imaging.

Supplementary Information The online version contains supplementary material available at https://doi.org/10.1007/s12265-021-10103-1.

Acknowledgments This research project was conducted during a research internship as part of the Research Master Clinical Research at the Netherlands Institute for Health Sciences.

\section{Funding None.}

\section{Declarations}

Ethics approval and consent to participate All patients provided written informed consent for the procedure and the use of anonymous datasets for research purposes in alignment with the Dutch Medical Research Act. Ethical approval for this study was waived by the Institutional Review Board of the Erasmus Medical Center because of the retrospective nature of the clinically collected data.

Disclosures Joost Daemen received institutional research support from Astra Zeneca, Abbott Vascular, Boston Scientific, Acist Medical, Medtronic, Pie Medical, ReCor medical, and PulseCath and consultancy and speaker fees from Acist Medical, Boston Scientific, ReCor Medical,
Pie Medical, Medtronic, and PulseCath. Nicolas M van Mieghem received research grant support from Edwards, Medtronic, Abbott, Boston Scientific, PulseCath, Acist Medical, and Essential Medical. Tara Neleman received institutional research grant support from Acist Medical.

Open Access This article is licensed under a Creative Commons Attribution 4.0 International License, which permits use, sharing, adaptation, distribution and reproduction in any medium or format, as long as you give appropriate credit to the original author(s) and the source, provide a link to the Creative Commons licence, and indicate if changes were made. The images or other third party material in this article are included in the article's Creative Commons licence, unless indicated otherwise in a credit line to the material. If material is not included in the article's Creative Commons licence and your intended use is not permitted by statutory regulation or exceeds the permitted use, you will need to obtain permission directly from the copyright holder. To view a copy of this licence, visit http://creativecommons.org/licenses/by/4.0/.

\section{References}

1. Mori, H., Torii, S., Kutyna, M., Sakamoto, A., Finn, A. V., \& Virmani, R. (2018). Coronary artery calcification and its progression: What does it really mean? JACC Cardiovasc Imaging, 11(1), 127-142.

2. Hoffmann, U., Massaro, J. M., D'Agostino Sr., R. B., Kathiresan, S., Fox, C. S., \& O'Donnell, C. J. (2016). Cardiovascular Event prediction and risk reclassification by coronary, aortic, and valvular calcification in the Framingham Heart Study. J Am Heart Assoc, 5(2).

3. Greenland, P., LaBree, L., Azen, S. P., Doherty, T. M., \& Detrano, R. C. (2004). Coronary artery calcium score combined with framingham score for risk prediction in asymptomatic individuals. [doi: 10.1001/jama.291.2.210]. JAMA, 291(2), 210-215.

4. Detrano, R., Guerci, A. D., Carr, J. J., Bild, D. E., Burke, G., Folsom, A. R., et al. (2008). Coronary calcium as a predictor of coronary events in four racial or ethnic groups. $N$ Engl J Med, 358(13), 1336-1345. 
5. Agatston, A. S., Janowitz, W. R., Hildner, F. J., Zusmer, N. R., Viamonte Jr., M., \& Detrano, R. (1990). Quantification of coronary artery calcium using ultrafast computed tomography. $J$ Am Coll Cardiol, 15(4), 827-832.

6. LaMonte, M. J., FitzGerald, S. J., Church, T. S., Barlow, C. E., Radford, N. B., Levine, B. D., et al. (2005). Coronary artery calcium score and coronary heart disease events in a large cohort of asymptomatic men and women. [doi: 10.1093/aje/kwi228]. American Journal of Epidemiology, 162(5), 421-429.

7. Peters, S. A., Bakker, M., den Ruijter, H. M., \& Bots, M. L. (2012). Added value of $\mathrm{CAC}$ in risk stratification for cardiovascular events: a systematic review. Eur J Clin Invest, 42(1), 110-116.

8. Kobayashi, Y., Okura, H., Kume, T., Yamada, R., Kobayashi, Y., Fukuhara, K., et al. (2014). Impact of target lesion coronary calcification on stent expansion. Circ J, 78(9), 2209-2214.

9. Fujii, K., Carlier, S. G., Mintz, G. S., Yang, Y. M., Moussa, I., Weisz, G., et al. (2005). Stent underexpansion and residual reference segment stenosis are related to stent thrombosis after sirolimus-eluting stent implantation: an intravascular ultrasound study. J Am Coll Cardiol, 45(7), 995-998.

10. Fujii, K., Mintz, G. S., Kobayashi, Y., Carlier, S. G., Takebayashi, H., Yasuda, T., et al. (2004). Contribution of stent underexpansion to recurrence after sirolimus-eluting stent implantation for in-stent restenosis. Circulation, 109(9), 1085-1088.

11. Mintz, G. S., Popma, J. J., Pichard, A. D., Kent, K. M., Satler, L. F., Chuang, Y. C., et al. (1995). Patterns of calcification in coronary artery disease. A statistical analysis of intravascular ultrasound and coronary angiography in 1155 lesions. Circulation, 91(7), 1959-1965.

12. Wang, X., Matsumura, M., Mintz, G. S., Lee, T., Zhang, W., Cao, Y., et al. (2017). In vivo calcium detection by comparing optical coherence tomography, intravascular ultrasound, and angiography. JACC Cardiovasc Imaging, 10(8), 869-879.

13. Shalev, R., Bezerra, H. G., Ray, S., Prabhu, D., \& Wilson, D. L. (2016). Classification of calcium in intravascular OCT images for the purpose of intervention planning. Proc SPIE Int Soc Opt Eng, 9786.

14. Lee, J., Hwang, Y. N., Kim, G. Y., Kwon, J. Y., \& Kim, S. M. (2019). Automated classification of dense calcium tissues in grayscale intravascular ultrasound images using a deep belief network. BMC Med Imaging, 19(1), 103.

15. Nair, A., Margolis, M. P., Kuban, B. D., \& Vince, D. G. (2007). Automated coronary plaque characterisation with intravascular ultrasound backscatter: ex vivo validation. EuroIntervention, 3(1), 113-120.

16. Liu, S., Neleman, T., Hartman, E. M. J., Ligthart, J. M. R., Witberg, K. T., van der Steen, A. F. W., et al. (2020). Automated quantitative assessment of coronary calcification using intravascular ultrasound. Ultrasound Med Biol, 46(10), 2801-2809.

17. Mintz, G. S., Nissen, S. E., Anderson, W. D., Bailey, S. R., Erbel, R., Fitzgerald, P. J., et al. (2001). American College of Cardiology Clinical Expert Consensus Document on Standards for Acquisition, Measurement and Reporting of Intravascular Ultrasound Studies (IVUS). A report of the American College of Cardiology Task Force on Clinical Expert Consensus Documents. J Am Coll Cardiol, 37(5), 1478-1492.

18. Garcia-Garcia, H. M., McFadden, E. P., Farb, A., Mehran, R., Stone, G. W., Spertus, J., et al. (2018). Standardized end point definitions for coronary intervention trials: The Academic Research Consortium-2 Consensus Document. Circulation, 137(24), 2635-2650.

19. New, S. E., Goettsch, C., Aikawa, M., Marchini, J. F., Shibasaki, M., Yabusaki, K., et al. (2013). Macrophage-derived matrix vesicles: an alternative novel mechanism for microcalcification in atherosclerotic plaques. Circ Res, 113(1), 72-77.

20. Kelly-Arnold, A., Maldonado, N., Laudier, D., Aikawa, E., Cardoso, L., \& Weinbaum, S. (2013). Revised microcalcification hypothesis for fibrous cap rupture in human coronary arteries. Proc Natl Acad Sci U S A, 110(26), 10741-10746.

21. Copeland-Halperin, R. S., Baber, U., Aquino, M., Rajamanickam, A., Roy, S., Hasan, C., et al. (2018). Prevalence, correlates, and impact of coronary calcification on adverse events following PCI with newer-generation DES: Findings from a large multiethnic registry. Catheter Cardiovasc Interv, 91(5), 859-866.

22. Genereux, P., Redfors, B., Witzenbichler, B., Arsenault, M. P., Weisz, G., Stuckey, T. D., et al. (2017). Two-year outcomes after percutaneous coronary intervention of calcified lesions with drugeluting stents. Int J Cardiol, 231, 61-67.

23. Genereux, P., Madhavan, M. V., Mintz, G. S., Maehara, A., Palmerini, T., Lasalle, L., et al. (2014). Ischemic outcomes after coronary intervention of calcified vessels in acute coronary syndromes. Pooled analysis from the HORIZONS-AMI (Harmonizing Outcomes With Revascularization and Stents in Acute Myocardial Infarction) and ACUITY (Acute Catheterization and Urgent Intervention Triage Strategy) TRIALS. J Am Coll Cardiol, 63(18), 1845-1854.

24. Bourantas, C. V., Zhang, Y. J., Garg, S., Iqbal, J., Valgimigli, M., Windecker, S., et al. (2014). Prognostic implications of coronary calcification in patients with obstructive coronary artery disease treated by percutaneous coronary intervention: a patient-level pooled analysis of 7 contemporary stent trials. Heart, 100(15), 1158-1164.

25. Vliegenthart, R., Oudkerk, M., Hofman, A., Oei, H. H., van Dijck, W., van Rooij, F. J., et al. (2005). Coronary calcification improves cardiovascular risk prediction in the elderly. Circulation, 112(4), 572-577.

26. Taylor, A. J., Bindeman, J., Feuerstein, I., Cao, F., Brazaitis, M., \& O'Malley, P. G. (2005). Coronary calcium independently predicts incident premature coronary heart disease over measured cardiovascular risk factors: mean three-year outcomes in the Prospective Army Coronary Calcium (PACC) project. J Am Coll Cardiol, 46(5), 807-814.

27. McBride, C. B., Cheezum, M. K., Gore, R. S., Pathirana, I. N., Slim, A. M., \& Villines, T. C. (2013). Coronary Artery calcium testing in symptomatic patients: An issue of diagnostic efficiency. Curr Cardiovasc Imaging Rep, 6(3), 211-220.

28. van der Giessen, A. G., Gijsen, F. J., Wentzel, J. J., Jairam, P. M., van Walsum, T., Neefjes, L. A., et al. (2011). Small coronary calcifications are not detectable by 64 -slice contrast enhanced computed tomography. Int J Cardiovasc Imaging, 27(1), 143-152.

29. Stone, G. W., Maehara, A., Lansky, A. J., de Bruyne, B., Cristea, E., Mintz, G. S., et al. (2011). A prospective natural-history study of coronary atherosclerosis. $N$ Engl J Med, 364(3), 226-235.

30. Calvert, P. A., Obaid, D. R., O'Sullivan, M., Shapiro, L. M., McNab, D., Densem, C. G., et al. (2011). Association between IVUS findings and adverse outcomes in patients with coronary artery disease: the VIVA (VH-IVUS in Vulnerable Atherosclerosis) Study. JACC Cardiovasc Imaging, 4(8), 894-901.

31. Nair, A., Kuban, B. D., Tuzcu, E. M., Schoenhagen, P., Nissen, S. E., \& Vince, D. G. (2002). Coronary plaque classification with intravascular ultrasound radiofrequency data analysis. Circulation, 106(17), 2200-2206.

32. Lee, J., Prabhu, D., Kolluru, C., Gharaibeh, Y., Zimin, V. N., Bezerra, H. G., et al. (2019). Automated plaque characterization using deep learning on coronary intravascular optical coherence tomographic images. Biomed Opt Express, 10(12), 6497-6515.

33. Oemrawsingh, R. M., Cheng, J. M., García-García, H. M., van Geuns, R. J., de Boer, S. P., Simsek, C., et al. (2014). Near-infrared spectroscopy predicts cardiovascular outcome in patients with coronary artery disease. J Am Coll Cardiol, 64(23), 2510-2518.

Publisher's Note Springer Nature remains neutral with regard to jurisdictional claims in published maps and institutional affiliations. 